\title{
Coherent States for Tremblay-Turbiner-Winternitz Potential $^{\star}$
}

Yusuf SUCU and Nuri UNAL

Department of Physics, Faculty of Science, Akdeniz University, 07058 Antalya, Turkey

E-mail: ysucu@akdeniz.edu.tr,nuriunal@akdeniz.edu.tr

Received July 31, 2012, in final form November 28, 2012; Published online December 01, 2012

http://dx.doi.org/10.3842/SIGMA.2012.093

\begin{abstract}
In this study, we construct the coherent states for a particle in the TremblayTurbiner-Winternitz potential by finding the conserved charge coherent states of the four harmonic oscillators in the polar coordinates. We also derive the energy eigenstates of the potential and show that the center of the coherent states follow the classical orbits of the particle.
\end{abstract}

Key words: Tremblay-Turbiner-Winternitz potential; generalized harmonic oscillator; noncentral potential; coherent state

2010 Mathematics Subject Classification: 81R30; 81Q05; 81Q80; 81 S99

\section{Introduction}

The coherent states were derived for the one-dimensional linear harmonic oscillator by Schrödinger [11] and used in the quantum theory of electrodynamics in 1963 and were recognized as the Glauber states $[4,5]$. The coherent states are particle like localized, non-dispersive solutions of the linear Schrödinger equation. In these states, the probability density is a time dependent Gaussian wave packet and the center of the packet follows the classical trajectory of the particle. Recently, the coherent states were constructed for the Kepler problem by transforming it into four harmonic oscillators evolving in a parametric-time [17]. This technique has been applied to derive the coherent states for a particle in the Morse potential [18], 5-dimensional Coulomb potential [21] and the non-central generalized MIC-Kepler potential [20]. For the coherent states, the expectation values of the position and momenta give the classical trajectories. Furthermore, the stationary quantum eigenstates and corresponding eigenvalues may be derived from the coherent states. Therefore, they are very important to discuss the relation between classical and quantum mechanics of a system.

Two important properties of a physical system in classical and quantum mechanics are the exact solvability and superintegrability. Some time ago, Tempesta, Turbiner and Winternitz conjectured that for two dimensional systems, all maximally superintegrable systems are exactly solvable [13]. Recently, in two dimensional plane, the following Hamiltonian has been proposed by Tremblay, Turbiner and Winternitz

$$
H=p_{r}^{2}+\frac{p_{\theta}^{2}}{r^{2}}+\omega^{2} r^{2}+\frac{1}{r^{2}}\left(\frac{\alpha k^{2}}{\sin ^{2} k \theta}+\frac{\beta k^{2}}{\cos ^{2} k \theta}\right), \quad 0 \leqslant \theta \leqslant \frac{\pi}{2 k} \quad \text { and } \quad 0 \leqslant r<\infty,
$$

where $\omega$ is the angular frequency of the oscillators, $\alpha$ and $\beta$ are nonnegative constants and $k$ is constant. The Schrödinger equation has been exactly solved for this Hamiltonian [12]. It includes all known examples of the two dimensional superintegrable systems for $k=1,2,3[2,3,9,15,25]$

${ }^{\star}$ This paper is a contribution to the Special Issue "Superintegrability, Exact Solvability, and Special Functions". The full collection is available at http://www.emis.de/journals/SIGMA/SESSF2012.html 
or $\alpha=0$ [1]. The quantum superintegrability of the system has been also discussed for odd $k$ values [10]. The conjecture about the superintegrability of the system has been supported by showing that for the classical version of the system all bounded trajectories are closed for all integer and rational values of the constant $k$ [14]. It has been proven that such systems in classical mechanics are superintegrable and supported the conjecture that all the orbits are closed for all rational values of $k$ [8]. Also, in order to present a constructive proof for the quantum superintegrable systems for all rational values of $k$, the canonical operator method has been applied [7]. In this connection, the superintegrability of a system is related into, in classical sense, the presence of closed and periodic orbits and, in quantum mechanics sense, the presence of a non-Abelian algebra for its integrals of motion and degenerate energy levels.

The $k=1$ case of equation (1) corresponds one of the four maximally superintegrable Smorodinsky-Winternitz potentials for which coherent states have been constructed $[16,19,23]$. The symmetry algebra of the system in $k=1$ case is quadratic, but, for other integer and rational values of $k$, these systems are separable in only one coordinate frame and the symmetry algebra is not quadratic. In classical and quantum sense, the integrability properties of the system have been investigated, in detail, for the other values of $k$, but the coherent states hasn't been discussed so far. Since the coherent states are the macrostates as a superposition of microstates of a system, they give both the classical and quantum behavior of the system. Therefore, it is interesting to generalize the coherent states and to find the expectation values of a system in equation (1) for the all integer and rational values of $k$.

The aim of this study is to construct the coherent states for a particle in a potential given by equation (1). In Section 2 we first discuss the mapping of the two dimensional system into four dimensional system in the harmonic oscillator potential, and the second, construct the coherent states for the four harmonic oscillators and the third, find the coherent states for the Hamiltonian $H$. We also derive the energy eigenstates of the system in polar coordinates. Section 3 we find the expectation values of radial and angular coordinates of the particle in the Tremblay-Turbiner-Winternitz (TTW) potential between the coherent states of the system. Section 4 is our conclusions.

\section{Coherent states for Tremblay-Turbiner-Winternitz potential}

To derive the coherent states for the TTW potential, we start by writing the action $A$ in the following way

$$
A=\int L d t=\int\left(p_{r} \frac{d r}{d t}+p_{\theta} \frac{d \theta}{d t}-H\right) d t
$$

where $p_{r}$ and $p_{\theta}$ are the radial and angular momenta of a particle in the two dimensional space with the following line element

$$
d s^{2}=(d r)^{2}+r^{2}(d \theta)^{2} .
$$

The Hamiltonian $H$ is similar to the radial Hamiltonian of the four harmonic oscillators. We change the angular coordinate $\theta$ as $\Theta=k \theta$, with $0 \leqslant \Theta \leqslant \frac{\pi}{2}$. Then the Hamiltonian becomes

$$
H=\left(p_{r}^{2}+\frac{k^{2} \mathbf{L}^{2}}{r^{2}}\right)
$$

where the constant of motion $\mathbf{L}^{2}$ is given as

$$
\mathbf{L}^{2}=\left[p_{\Theta}^{2}+\left(\frac{\alpha}{\sin ^{2} \Theta}+\frac{\beta}{\cos ^{2} \Theta}\right)\right] .
$$


Therefore, to construct the coherent states of the system, we first reduce the system into the $k=1$ case and take the coherent states of the Smorodinsky-Winternitz potential. We finally replace the quantum numbers corresponding to $\mathbf{L}^{2}$ by $k^{2} \mathbf{L}^{2}$.

In order to represent these potentials as the kinetic energy contribution of the angular momenta of the particle on $S^{3}$, we first introduce the cartesian coordinates of the particle $u$ and $v$ as

$$
(u, v)=\sqrt{\omega} r(\cos \Theta, \sin \Theta),
$$

with the condition of $u, v \geq 0$ which corresponds to represent the system as two isotropic polar harmonic oscillators with centrifugal barrier $\left(\frac{\alpha}{u^{2}}+\frac{\beta}{v^{2}}\right)$. In polar coordinates $\left(\frac{\partial}{\partial u}, \frac{\partial}{\partial v}\right) \Psi$ is replaced by $\left(\frac{\partial}{\partial u}+\frac{1}{2 u}, \frac{\partial}{\partial v}+\frac{1}{2 v}\right) \frac{\Psi}{\sqrt{u v}}$ and centrifugal barrier $\left(\frac{\alpha}{u^{2}}+\frac{\beta}{v^{2}}\right)$ become $\left(\frac{\alpha+1 / 4}{u^{2}}+\frac{\beta+1 / 4}{v^{2}}\right)$. By using this prescription we get the following Hamiltonian in dimensionless units

$$
\widetilde{H}=p_{u}^{2}+p_{v}^{2}+u^{2}+v^{2}+\frac{\alpha+\frac{1}{4}}{u^{2}}+\frac{\beta+\frac{1}{4}}{v^{2}} .
$$

Second, we introduce two dummy angles $\phi$ and $\psi$ by the Lagrange multipliers without changing the dynamics of the particle. We also change $p_{r}$ and $p_{\Theta}$ as the radial and angular momenta of a particle in four dimensional space with the the line element

$$
d s^{2}=(d u)^{2}+(d v)^{2}+u^{2}(d \phi)^{2}+v^{2}(d \psi)^{2} .
$$

Thus the Lagrangian $L$ becomes

$$
\widetilde{L}=p_{u} \frac{d u}{d \omega t}+p_{v} \frac{d v}{d \omega t}+\frac{d \phi}{d \omega t}\left(p_{\phi}-\sqrt{\alpha+\frac{1}{4}}\right)+\frac{d \psi}{d \omega t}\left(p_{\psi}-\sqrt{\beta+\frac{1}{4}}\right)-\widetilde{H}
$$

where the Hamiltonian $\widetilde{H}$ is rewritten as

$$
\widetilde{H}=p_{u}^{2}+p_{v}^{2}+u^{2}+v^{2}+\frac{p_{\phi}^{2}}{u^{2}}+\frac{p_{\psi}^{2}}{v^{2}} .
$$

The coherent states are given in configuration space [22] $u_{i}, v_{i}$ with $i=1,2$ as

$$
\widetilde{\Psi}_{\lambda_{i}(t)}\left(u_{i}\right)=N \prod_{i=1}^{4} e^{-4 i \omega t} e^{-\frac{1}{2} u^{2}+\kappa_{i}(t) u_{i}} e^{-\frac{1}{2} v^{2}+\lambda_{i}(t) v_{i}} .
$$

Here we use the natural units $\hbar=1$ and the complex eigenvalues of the harmonic oscillators lowering operators $\kappa_{i}(t)$ and $\lambda_{i}(t)$ are given as

$$
\kappa_{i}(t)=\kappa_{i}(0) e^{-2 i \omega t}, \quad \lambda_{i}(t)=\lambda_{i}(0) e^{-2 i \omega t} .
$$

In polar coordinates $(u, \phi)$ and $(v, \psi)$ the coherent states are given as

$$
\widetilde{\Psi}_{\lambda_{i}(t)}\left(u_{i}\right)=N e^{-4 i \omega t} e^{-\frac{1}{2} u^{2}} e^{u\left(\frac{\kappa_{1}(t)-i \kappa_{2}(t)}{2} e^{i \phi}+\frac{\kappa_{1}(t)+i \kappa_{2}(t)}{2} e^{-i \phi}\right)} e^{v\left(\frac{\lambda_{3}(t)-i \lambda_{4}(t)}{2} e^{i \psi}+\frac{\lambda_{1}(t)+i \lambda_{2}(t)}{2} e^{-i \psi}\right) .} .
$$

If we expand the exponential expressions into the power series we get

$$
\begin{aligned}
\widetilde{\Psi}_{\lambda_{i}(t)}\left(u_{i}\right)= & N e^{-4 i \omega t} e^{-\frac{1}{2}\left(u^{2}+v^{2}\right)} \sum_{\mu_{1}=-\infty}^{+\infty}[K(0)]^{\mu_{1}} J_{\mu_{1}}\left(\sqrt{u^{2}(-i \kappa(t))^{2}}\right) \exp i \mu_{1} \phi \\
& \times \sum_{\mu_{2}=-\infty}^{+\infty}[\Lambda(0)]^{\mu_{2}} J_{\mu_{2}}\left(2 \sqrt{v^{2}((-i \lambda(t)))^{2}}\right) \exp i \mu_{2} \psi,
\end{aligned}
$$


where $K(0), \Lambda(0)$ and $\kappa(t), \lambda(t)$ are defined as

$$
\begin{array}{lll}
\kappa(t)=\sqrt{\left[\lambda_{2}(t)+i \lambda_{1}(t)\right]\left[\lambda_{2}(t)-i \lambda_{1}(t)\right]}, & \lambda(t)=\sqrt{\left[\lambda_{4}(t)+i \lambda_{3}(t)\right]\left[\lambda_{4}(t)-i \lambda_{3}(t)\right]}, \\
K(0)=\sqrt{\left[\lambda_{2}(t)+i \lambda_{1}(t)\right] /\left[\lambda_{2}(t)-i \lambda_{1}(t)\right]}, & \Lambda(0)=\sqrt{\left[\lambda_{4}(t)+i \lambda_{3}(t)\right] /\left[\lambda_{4}(t)-i \lambda_{3}(t)\right]} .
\end{array}
$$

We notice that in equation (2) the eigenvalues $K(0)$ and $\Lambda(0)$ are time independent and they correspond to the conserved quantities (charges) $L_{12}$ and $L_{34}$ or $p_{\phi}$ and $p_{\psi}$. Therefore, the eigenvalues corresponding to the polar parts of the four oscillators are time dependent.

To derive the coherent states of the physical particle $\widetilde{\Psi}^{\text {phys }}$ we consider elimination of the dummy coordinates $\phi$ and $\psi$. There are two methods of elimination: in wave function formalism we consider physical eigenvalues of corresponding conjugate momenta $\widehat{p}_{\phi}$ and $\widehat{p}_{\psi}$ by taking care of that these dummy coordinates are not cyclic and the conjugate momenta have continuous eigenvalues

$$
\widehat{p}_{\phi} \widetilde{\Psi}^{\text {phys }}=\sqrt{\alpha+\frac{1}{4}} \widetilde{\Psi}^{\text {phys }}, \quad \widehat{p}_{\psi} \widetilde{\Psi}^{\text {phys }}=\sqrt{\beta+\frac{1}{4}} \widetilde{\Psi}^{\text {phys }} .
$$

In the path integration formalism, we integrate over all the possible final values of these variables. Then $\widetilde{\Psi}^{\text {phys }}$ becomes

$$
\begin{aligned}
\widetilde{\Psi}_{\lambda_{i}(t)}^{\mathrm{phys}}\left(u_{i}\right)= & N e^{-4 i \omega t} e^{-\frac{1}{2}\left(u^{2}+v^{2}\right)}\left[K(0) e^{i \phi}\right]^{p_{\phi}}\left[\Lambda(0) e^{i \psi}\right]^{p_{\psi}} \\
& \times J_{p_{\phi}}\left(\sqrt{u^{2}(-i \kappa(t))^{2}}\right) J_{p_{\psi}}\left(\sqrt{v^{2}(-i \lambda(t))^{2}}\right) .
\end{aligned}
$$

We parameterize the product of two Bessel functions as

$$
\begin{aligned}
& J_{p_{\phi}}\left(\sqrt{u^{2}(-i \kappa(t))^{2}}\right) J_{p_{\psi}}\left(\sqrt{v^{2}(-i \lambda(t))^{2}}\right) \\
& \quad=J_{p_{\phi}}\left(\sqrt{u^{2}+v^{2}} \cos \Theta(-i) \sqrt{\kappa^{2}+\lambda^{2}} \cos \Phi\right) J_{p_{\psi}}\left(\sqrt{u^{2}+v^{2}} \sin \Theta(-i) \sqrt{\kappa^{2}+\lambda^{2}} \sin \Phi\right),
\end{aligned}
$$

where

$$
(u, v)=\sqrt{u^{2}+v^{2}}(\sin \Theta, \cos \Theta), \quad(\kappa, \lambda)=\sqrt{\kappa^{2}+\lambda^{2}}(\sin \Phi, \cos \Phi) .
$$

We write the product of two Bessel functions $J_{p_{\phi}}$ and $J_{p_{\psi}}$ in terms of one Bessel function [24]

$$
\begin{aligned}
& J_{p_{\phi}}\left(-i \sqrt{u^{2}+v^{2}} \sin \Theta \sqrt{\kappa^{2}+\lambda^{2}} \sin \Phi\right) J_{p_{\psi}}\left(-i \sqrt{u^{2}+v^{2}} \cos \Theta \sqrt{\kappa^{2}+\lambda^{2}} \cos \Phi\right) \\
& =\sum_{l_{1}=0}^{\infty} N_{l_{1}}^{\left(p_{\phi}, p_{\psi}\right)} \frac{J_{2 l_{1}+p_{\phi}+p_{\psi}+1}\left(-i \sqrt{u^{2}+v^{2}} \sqrt{\kappa^{2}+\lambda^{2}}\right)}{-2 i \sqrt{u^{2}+v^{2}} \sqrt{\kappa^{2}+\lambda^{2}}} d_{p_{\phi}, p_{\psi}}^{l_{1}}(\cos 2 \Phi) d_{p_{\phi}, p_{\psi}}^{l_{1}}(\cos 2 \Theta),
\end{aligned}
$$

where the constant $N_{l_{1}}^{\left(p_{\phi}, p_{\psi}\right)}$ is

$$
N_{l_{1}}^{\left(p_{\phi}, p_{\psi}\right)}=\frac{i(-1)^{l_{1}} l_{1} ! \Gamma\left(p_{\phi}+p_{\psi}+l_{1}+1\right)}{\Gamma\left(p_{\phi}+l_{1}+1\right) \Gamma\left(p_{\phi}+l_{1}+1\right)}
$$

and the angular wave functions $d_{p_{\phi}, p_{\psi}}^{l_{1}}(\cos 2 \Theta)$ are defined in terms of Jacobi polynomials $P_{p_{\phi}, p_{\psi}}^{l_{1}}(\cos 2 \Theta)$ as

$$
d_{p_{\phi}, p_{\psi}}^{l_{1}}(\cos 2 \Theta)=(\sin \Theta)^{p_{\phi}}(\cos \Theta)^{p_{\psi}} P_{p_{\phi}, p_{\psi}}^{l_{1}}(\cos 2 \Theta) .
$$

In previous equation the generalized conserved charge coherent states are parameterized by the time dependent eigenvalues

$$
\sqrt{\kappa^{2}+\lambda^{2}}=\sqrt{\kappa^{2}(0)+\lambda^{2}(0)} e^{-2 i \omega t},
$$


and the time independent eigenvalues

$$
\sqrt{\kappa^{2} / \lambda^{2}}=\sqrt{\kappa^{2}(0) / \lambda^{2}(0)}
$$

Here $\sqrt{\kappa^{2} / \lambda^{2}}$ corresponds to the conserved charge related to $L^{2}$. In equation (3) the time independent phase factors are omitted.

Then except some constant phase factors $\widetilde{\Psi}^{\text {phys }}$ becomes

$$
\begin{aligned}
\widetilde{\Psi}_{\sqrt{\kappa^{2}+\lambda^{2}}}^{\text {phys }}(r, \Theta)= & \frac{N}{4} e^{-4 i \omega t} e^{-\frac{1}{2} \omega r^{2}} \sum_{l_{1}=0}^{\infty} N_{l_{1}}^{\left(p_{\phi}, p_{\psi}\right)} d_{p_{\phi}, p_{\psi}}^{l_{1}}(\cos 2 \Phi) \\
& \times \frac{J_{2 l_{1}+p_{\phi}+p_{\psi}+1}\left(2 \sqrt{\omega r^{2}} \frac{\sqrt{(-i)^{2}\left(\kappa^{2}+\lambda^{2}\right)}}{2}\right)}{\sqrt{\omega r^{2}} \frac{\sqrt{(-i)^{2}\left(\kappa^{2}+\lambda^{2}\right)}}{2}} d_{p_{\phi}, p_{\psi}}^{l_{1}}(\cos 2 \Theta) .
\end{aligned}
$$

Here we expand the Bessel functions in terms of Laguerre functions [6]. The conserved charge coherent states of the four oscillators are given as

$$
\begin{aligned}
\widetilde{\Psi}_{\sqrt{\kappa^{2}+\lambda^{2}}}^{\text {phys }}(r, \Theta)= & \frac{N}{4} \sum_{l_{1}=0}^{\infty} N_{l_{1}}^{\left(p_{\phi}, p_{\psi}\right)} d_{p_{\phi}, p_{\psi}}^{l_{1}}(\cos 2 \Phi)(\sin k \theta)^{p_{\phi}}(\cos k \theta)^{p_{\psi}} P_{p_{\phi}, p_{\psi}}^{l_{1}}\left(2 \sin ^{2} k \theta-1\right) \\
& \times \sum_{n_{r}=0}^{\infty} \frac{e^{\frac{\left(|\kappa|^{2}+|\lambda|^{2}\right)}{4}} e^{-4 i \omega t\left(\frac{-\left(\kappa^{2}+\lambda^{2}\right)}{4}\right)}}{\Gamma\left(n_{r}+2 l_{1}+p_{\phi}+p_{\psi}+2\right)} \\
& \times e^{-\frac{\left(2 l_{1}+p_{\phi}+p_{\psi}+1\right)}{2} \omega r^{2}}\left(\omega r^{2}\right)^{\frac{\left(2 l_{1}+p_{\phi}+p_{\psi}+1\right)-1}{2}} L_{n_{r}}^{\left(2 l_{1}+p_{\phi}+p_{\psi}+1\right)}\left(\omega r^{2}\right) .
\end{aligned}
$$

In order to find the coherent states of a system described by TTW Hamiltonian $H$ for the terms under the $n_{r}$ summation, we first replace $\left(2 l_{1}+p_{\phi}+p_{\psi}+1\right)$ by $\left(2 l_{1}+p_{\phi}+p_{\psi}+1\right) k$ and multiply the wave function $\widetilde{\Psi}_{\sqrt{\kappa^{2}+\lambda^{2}}}^{\text {phys }}(r, \Theta)$ by $\sqrt{\omega r^{2} \sin \Theta \cos \Theta}$.

Then, we find the coherent states of the TTW system as

$$
\begin{aligned}
& \Psi_{\sqrt{\kappa^{2}+\lambda^{2}}}^{\mathrm{phys}}(r, \theta)= \frac{N}{4} \sum_{l_{1}=0}^{\infty} N_{l_{1}}^{\left(p_{\phi}, p_{\psi}\right)} d_{p_{\phi}, p_{\psi}}^{l_{1}}(\cos 2 \Phi)(\sin k \theta)^{p_{\phi}+\frac{1}{2}}(\cos k \theta)^{p_{\psi}+\frac{1}{2}} \\
& \times P_{p_{\phi}, p_{\psi}}^{l_{1}}\left(2 \sin ^{2} k \theta-1\right) \sum_{n_{r}=0}^{\infty} \frac{e^{\frac{\left(|\kappa|^{2}+|\lambda|^{2}\right)}{4}} e^{-4 i \omega t\left(\frac{-\left(\kappa^{2}+\lambda^{2}\right)}{4}\right)}}{\Gamma\left[n_{r}+\left(2 l_{1}+p_{\phi}+p_{\psi}+1\right) k+1\right]} \\
& \times e^{-\frac{1}{2} \omega r^{2}}\left(\omega r^{2}\right)^{\frac{\left(2 l_{1}+p_{\phi}+p_{\psi}+1\right) k}{4}+\frac{n_{r}}{2}} \\
&
\end{aligned}
$$

Here time independent energy eigenstates are given as

$$
\begin{aligned}
\Psi_{n_{r}, l_{1}}^{\mathrm{phys}}(r, \theta)= & (\sin k \theta)^{p_{\phi}+\frac{1}{2}}(\cos k \theta)^{p_{\psi}+\frac{1}{2}} P_{p_{\phi}, p_{\psi}}^{l_{1}}\left(2 \sin ^{2} k \theta-1\right) \\
& \times e^{-\frac{1}{2} \omega r^{2}}\left(\omega r^{2}\right)^{\frac{\left(2 l_{1}+p_{\phi}+p_{\psi}+1\right) k}{2}} L_{n_{r}}^{\left(2 l_{1}+p_{\phi}+p_{\psi}+1\right) k}\left(\omega r^{2}\right),
\end{aligned}
$$

and the energy eigenvalues are

$$
E=2\left(2 l_{1}+p_{\phi}+p_{\psi}+1\right) k+2 n_{r} .
$$

The spectrum is degenerate for integer and rational values of $k$. So, the classical orbits are closed for these $k$ cases. The energy eigenstates and eigenvalues are the same with the result given in [12]. 


\section{Expectation values}

For the coherent states of the isotropic four harmonic oscillators the expectation values in the configuration space $\left\langle u_{i}\right\rangle,\left\langle v_{i}\right\rangle$ are given as

$$
\left\langle u_{i}\right\rangle_{t}=\frac{\kappa_{i}(t)+\kappa_{i}^{*}(t)}{2}=\left|\kappa_{i}(0)\right| \cos \left(2 \omega t-\varphi_{i}\right),
$$

and

$$
\left\langle v_{i}\right\rangle_{t}=\frac{\lambda_{i}(t)+\lambda_{i}^{*}(t)}{2}=\left|\lambda_{i}(0)\right| \cos \left(2 \omega t-\chi_{i}\right)
$$

where $\varphi_{i}$ and $\chi_{i}$ are the phases of $\kappa_{i}(0)$ and $\lambda_{i}(0)$. Then for the square of radial coordinate $\left\langle u_{i}\right\rangle_{t}^{2}$ the expectation value is given as

$$
\langle u\rangle_{t}^{2}=\left\langle u_{1}\right\rangle^{2}+\left\langle u_{2}\right\rangle^{2}=\frac{\left|\kappa_{1}(0)\right|^{2}+\left|\kappa_{2}(0)\right|^{2}}{2}+\frac{|\kappa(0)|^{2}}{2} \cos (4 \omega t-2 \varphi) .
$$

Here we have neglected the uncertainties and the constants, $\kappa(0)$ and $2 \varphi$ are given as

$$
\left.|\kappa(0)|^{2}=\left[\left|\kappa_{1}(0)\right|^{4}+\left|\kappa_{2}(0)\right|^{4}+2\left|\kappa_{1}(0) \kappa_{2}(0)\right|^{2} \cos 2 \varphi_{1}-\varphi_{2}\right)\right]^{\frac{1}{2}},
$$

and

$$
\tan 2 \varphi=\frac{\left|\kappa_{1}(0)\right|^{2} \sin 2 \varphi_{1}+\left|\kappa_{1}(0)\right|^{2} \sin 2 \varphi_{2}}{\left|\kappa_{1}(0)\right|^{2} \cos 2 \varphi_{1}+\left|\kappa_{1}(0)\right|^{2} \cos 2 \varphi_{2}}
$$

In order to derive the expectation values between the conserved charge coherent states, we evaluate the angular momentum $L_{12}$ as

$$
L_{12}=-\frac{\left(\kappa_{1}(t) \kappa_{2}^{*}(t)-\kappa_{2}(t) \kappa_{1}^{*}(t)\right)}{2 i}=-\left|\kappa_{1}(0) \kappa_{2}(0)\right| \sin \left(\varphi_{1}-\varphi_{2}\right) .
$$

We fix the value of $L_{12}$ as $\sqrt{\alpha+\frac{1}{4}}$. Then

$$
\frac{\cos 2\left(\varphi_{1}-\varphi_{2}\right)}{2}=1-\frac{2\left(\alpha+\frac{1}{4}\right)}{\left|\kappa_{1}(0) \kappa_{2}(0)\right|^{2}}
$$

and

$$
|\kappa(0)|^{2}=\left[\left(\left|\kappa_{1}(0)\right|^{2}+\left|\kappa_{2}(0)\right|^{2}\right)^{2}-4\left(\alpha+\frac{1}{4}\right)\right]^{\frac{1}{2}} .
$$

As a result of this, for $k=1,\langle u\rangle_{t}^{2}$ is given as

$$
\begin{aligned}
\langle u\rangle_{t}^{2} & =\left\langle u_{1}\right\rangle^{2}+\left\langle u_{2}\right\rangle^{2} \\
& =\frac{\left|\kappa_{1}(0)\right|^{2}+\left|\kappa_{2}(0)\right|^{2}}{2}+\left[\frac{\left(\left|\kappa_{1}(0)\right|^{2}+\left|\kappa_{2}(0)\right|^{2}\right)^{2}}{4}-\left(\alpha+\frac{1}{4}\right)\right]^{\frac{1}{2}} \cos (4 \omega t-2 \varphi) .
\end{aligned}
$$

In the similar way $\langle v\rangle_{t}^{2}$ is given as

$$
\begin{aligned}
\langle v\rangle_{t}^{2} & =\left\langle v_{1}\right\rangle^{2}+\left\langle v_{2}\right\rangle^{2} \\
& =\frac{\left|\lambda_{1}(0)\right|^{2}+\left|\lambda_{2}(0)\right|^{2}}{2}+\left[\frac{\left(\left|\lambda_{1}(0)\right|^{2}+\left|\lambda_{2}(0)\right|^{2}\right)^{2}}{4}-\left(\beta+\frac{1}{4}\right)\right]^{\frac{1}{2}} \cos (4 \omega t-2 \chi) .
\end{aligned}
$$


These are the expectation values of $\langle u\rangle_{t}^{2}$ and $\langle v\rangle_{t}^{2}$ between the conserved charge coherent states for the radial oscillators given by the Hamiltonian $\widetilde{H}$. In two-dimensional oscillator with polar coordinates $(u, \phi)$ the expectation value of unit vector $\langle\hat{\phi}\rangle$ also oscillates in time. For the reduction of dimension we fix the oscillations in $\langle\widehat{\phi}\rangle$ and take it as a constant unit vector.

To find the expectation value of radial coordinates we arrange the following product

$$
\begin{aligned}
{\left[\kappa_{1}^{2}(t)\right.} & \left.+\kappa_{2}^{2}(t)+\lambda_{1}^{2}(t)+\lambda_{2}^{2}(t)\right]\left[\kappa_{1}^{* 2}(t)+\kappa_{2}^{* 2}(t)+\lambda_{1}^{* 2}(t)+\lambda_{2}^{* 2}(t)\right] \\
= & {\left[\left|\kappa_{1}(0)\right|^{2}+\left|\kappa_{2}(0)\right|^{2}+\left|\lambda_{1}(0)\right|^{2}+\left|\lambda_{2}(0)\right|^{2}\right]^{2} } \\
& +\left[\kappa_{1}(0) \kappa_{2}^{*}(0)-\kappa_{2}(0) \kappa_{1}^{*}(0)\right]^{2}+\left[\lambda_{1}(0) \lambda_{2}^{*}(0)-\lambda_{2}(0) \lambda_{1}^{*}(0)\right]^{2} \\
& +\left[\kappa_{1}(0) \lambda_{1}^{*}(0)-\lambda_{1}(0) \kappa_{1}^{*}(0)\right]^{2}+\left[\kappa_{1}(0) \lambda_{2}^{*}(0)-\lambda_{2}(0) \kappa_{1}^{*}(0)\right]^{2} \\
& +\left[\kappa_{2}(0) \lambda_{1}^{*}(0)-\lambda_{1}(0) \kappa_{2}^{*}(0)\right]^{2}+\left[\kappa_{2}(0) \lambda_{2}^{*}(0)-\lambda_{2}(0) \kappa_{2}^{*}(0)\right]^{2} \\
= & 4\left(\frac{E}{\omega}\right)^{2}-4 \mathbf{L}^{2} .
\end{aligned}
$$

Then, for $k=1$ case, $\langle r\rangle_{t}^{2}$ becomes

$$
\omega\langle r\rangle_{t}^{2}=\left(\frac{E}{2 \omega}\right)+\left[\left(\frac{E}{2 \omega}\right)^{2}-\mathbf{L}^{2}\right]^{\frac{1}{2}} \sin 2(2 \omega t-\delta) .
$$

Here the constants of motion are defined as

$$
\frac{E}{\omega}=\frac{\left|\kappa_{1}(0)\right|^{2}+\left|\kappa_{2}(0)\right|^{2}+\left|\lambda_{1}(0)\right|^{2}+\left|\lambda_{2}(0)\right|^{2}}{2}
$$

and

$$
\cot 2 \delta=\frac{|\kappa(0)|^{2} \sin 2 \varphi+|\lambda(0)|^{2} \sin 2 \chi}{|\kappa(0)|^{2} \cos 2 \varphi+|\lambda(0)|^{2} \cos 2 \chi}
$$

In order to derive the expectation values for TTW Hamiltonian we replace $\mathbf{L}^{2}, \alpha+\frac{1}{2}$, and $\beta+\frac{1}{2}$ by $k^{2} \mathbf{L}^{2}, k\left(\alpha+\frac{1}{2}\right)$ and $k\left(\beta+\frac{1}{2}\right)$, respectively. Then, $\omega\langle r\rangle_{t}^{2}$ becomes

$$
\langle r\rangle_{t}^{2}=\frac{E}{2 \omega^{2}}+\left[\left(\frac{E}{2 \omega^{2}}\right)^{2}-\frac{A}{\omega^{2}}\right]^{\frac{1}{2}} \sin 4 \omega\left(t-t_{0}\right),
$$

where $A$ is $k^{2} \mathbf{L}^{2}$. The $\langle r\rangle_{t}$ shows the center of the coherent states following the classical trajectories of the particle in this TTW potential. It should be emphasized that the result on the radial coordinate of expectation value derived from the coherent states agrees with the result of [14] in which bounded trajectories are investigated for the integer and rational values of $k$.

The expectation value of $\sin \Theta$ may be derived from $\langle u\rangle_{t}^{2}$ and $\omega\langle r\rangle_{t}^{2}$ in equations (5) and (6) as

$$
\langle\sin \Theta\rangle^{2}=\left.\frac{\langle u\rangle_{t}^{2}}{\omega\langle r\rangle_{t}^{2}}\right|_{L_{12}^{2}=k\left(\alpha+\frac{1}{2}\right)} .
$$

\section{Conclusion}

In this study, we have constructed the coherent states for the Tremblay-Turbiner-Winternitz potential. One of the constants of motion is $k^{2} \mathbf{L}^{2}$. Therefore, for the case $k \neq 1$, we have integrated the equations of motion in polar coordinates by using the solutions for $k=1$ case, 
since the separability in this coordinates do not depend on the value of $k$. For $k=1$ case, the Schrödinger equation is also separable in cartesian coordinates $(u, v)$.

For the Tremblay-Turbiner-Winternitz potential, the coherent states correspond to the conserved charge coherent states of the four oscillators with the eigenvalues of $k \sqrt{\alpha+\frac{1}{4}}, k \sqrt{\beta+\frac{1}{4}}$ and $k^{2}\left[2 l_{1}\left(2 l_{1}+p_{\phi}+p_{\psi}+1\right)\right]$ of the operators $k L_{12}, k L_{34}$, and $k^{2}\left[p_{\Theta}^{2}+\left(\frac{\alpha}{\sin ^{2} \Theta}+\frac{\beta}{\cos ^{2} \Theta}\right)\right]$, respectively. The energy eigenvalues are given as by equation (4), and as it was shown previously in [12], the spectrum becomes degenerate for the integer and rational values of the parameter $k$. This result corresponds to the periodicity of the Hamiltonian: if the Hamiltonian has angular periodicity of $\frac{\pi m}{n}$ for any of two integers $m$ and $n$, then $k$ must be an integer or rational number. Furthermore, we have evaluated the expectation value of the radial coordinate $\langle r\rangle_{t}$, and shown that the center of the coherent states follow the classical trajectories of the particle in the Tremblay-Turbiner-Winternitz potential.

\section{Acknowledgements}

This work was supported by Akdeniz University, Scientific Research Projects Unit.

\section{References}

[1] Calogero F., Solution of a three-body problem in one dimension, J. Math. Phys. 10 (1969), 2191-2197.

[2] Evans N.W., Group theory of the Smorodinsky-Winternitz system, J. Math. Phys. 32 (1991), 3369-3375.

[3] Friš J., Mandrosov V., Smorodinsky Ya.A., Uhlír M., Winternitz P., On higher symmetries in quantum mechanics, Phys. Lett. 16 (1965), 354-356.

[4] Glauber R.J., Photon correlations, Phys. Rev. Lett. 10 (1963), 84-86.

[5] Glauber R.J., The quantum theory of optical coherence, Phys. Rev. 130 (1963), 2529-2539.

[6] Gradshteyn I.S., Ryzhik I.M., Table of integrals, series, and products, Academic Press, New York, 1980.

[7] Kalnins E.G., Kress J.M., Miller W., Superintegrability and higher order integrals for quantum systems, J. Phys. A: Math. Theor. 43 (2010), 265205, 21 pages, arXiv:1002.2665.

[8] Kalnins E.G., Miller W., Pogosyan G.S., Superintegrability and higher order constants for classical and quantum systems, Phys. Atomic Nuclei 74 (2011), 914-918, arXiv:0912.2278.

[9] Olshanetsky M.A., Perelomov A.M., Quantum integrable systems related to Lie algebras, Phys. Rep. 94 (1983), 313-404.

[10] Quesne C., Superintegrability of the Tremblay-Turbiner-Winternitz quantum Hamiltonians on a plane for odd k, J. Phys. A: Math. Theor. 43 (2010), 082001, 10 pages, arXiv:0911.4404.

[11] Schrödinger E., Der stetige Übergang von der Mikro- zur Makromechanik, Naturwissenschaften 14 (1926), 664-666.

[12] Tremblay F., Turbiner A.V., Winternitz P., An infinite family of solvable and integrable quantum systems on a plane, J. Phys. A: Math. Theor. 42 (2009), 242001, 10 pages, arXiv:0904.0738.

[13] Tempesta P., Turbiner A.V., Winternitz P., Exact solvability of superintegrable systems, J. Math. Phys. 42 (2001), 4248-4257, hep-th/0011209.

[14] Tremblay F., Turbiner A.V., Winternitz P., Periodic orbits for an infinite family of classical superintegrable systems, J. Phys. A: Math. Theor. 43 (2010), 015202, 14 pages, arXiv:0910.0299.

[15] Turbiner A., Hidden algebra of three-body integrable systems, Modern Phys. Lett. A 13 (1998), 1473-1483, solv-int/9805003.

[16] Unal N., Coherent states for Smorodinsky-Winternitz potentials, Cent. Eur. J. Phys. 7 (2009), 774-785.

[17] Unal N., Parametric time-coherent states for the hydrogen atom, Phys. Rev. A 63 (2001), 052105, 8 pages.

[18] Unal N., Parametric-time coherent states for Morse potential, Can. J. Phys. 80 (2002), 875-881.

[19] Unal N., Parametric-time coherent states for Smorodinsky-Winternitz potentials, J. Math. Phys. 48 (2007), 122107, 20 pages. 
[20] Unal N., Parametric-time coherent states for the generalized MIC-Kepler system, J. Math. Phys. 47 (2006), 122105, 15 pages.

[21] Unal N., Path integration and coherent states for the 5D hydrogen atom, in Fluctuating Paths and Fields, Editors W. Janke, A. Pelster, H.J. Schmidt, M. Bachmann, World Sci. Publ., River Edge, NJ, 2001, 73-81.

[22] Unal N., Quasi-coherent states for harmonic oscillator with time-dependent parameters, J. Math. Phys. 53 (2012), 012102, 8 pages.

[23] Unal N., Smorodinsky-Winternitz potentials: coherent, state approach, Phys. Atomic Nuclei 74 (2011), 1758-1769.

[24] Watson G.N., A treatise on the theory of Bessel functions, Cambridge University Press, London, 1922.

[25] Wolfes J., On the three-body linear problem with three-body interaction, J. Math. Phys. 15 (1974), 14201424 . 